\title{
Propuesta metodológica para diseñar productos más sostenibles en la etapa de producción
}

\author{
Natalia Muñoz-López \\ nataliam@unizar.es \\ Dpto. de Ingeniería de Diseño y Fabricación - EINA (Universidad de Zaragoza) C/ María de \\ Luna, 3 (50018) Zaragoza, España. \\ https://orcid.org/0000-0001-9643-6076 \\ José Luis Santolaya-Sáenz \\ Dpto. de Ingeniería de Diseño y Fabricación - EINA (Universidad de Zaragoza) C/ María de \\ Luna, 3 (50018) Zaragoza, España.
}

\section{Anna Biedermann}

Dpto. de Ingeniería de Diseño y Fabricación - EINA (Universidad de Zaragoza) C/ María de Luna, 3 (50018) Zaragoza, España.

Recepción:07 de abril del 2021 Aprobación:28 de mayo del 2021 Publicación: 06 de junio del 2021

\section{RESUMEN}

La eficiencia y la sostenibilidad son un reto para todo tipo de productos y procesos y actuar desde la actividad del diseño puede ayudar a alcanzarlo. En las últimas décadas, el Eco-diseño, centrado en el análisis de los impactos ambientales de un producto, ha evolucionado progresivamente hacia métodos que analizan simultáneamente las tres dimensiones de la sostenibilidad: medioambiental, económica y social.

Este trabajo presenta una propuesta metodológica para proyectar productos más sostenibles en la etapa de producción. Esta propuesta pretende facilitar a estudiantes de ingeniería el desarrollo metodológico de proyectos e investigaciones con criterios basados en la sostenibilidad. Se compone de tres fases principales: evaluación de la sostenibilidad aplicando la metodología Life Cycle Sustainability Assessment (LCSA), aplicación de estrategias teniendo en cuenta los requerimientos iniciales del producto y comparación final de diseños. Se aplica un enfoque ingenieril que define métricas ligadas a aspectos técnicos y selecciona un conjunto de indicadores cuantitativos adecuados para evaluar la sostenibilidad en cada una de sus dimensiones. Se selecciona la categoría de los trabajadores para evaluar los aspectos sociales.

La metodología se pone en práctica en dos casos de estudio: la fabricación de un contenedor isotermo y la producción de leche en una explotación de ganado vacuno. Se 
analizan los datos de inventario de todos los materiales, procesos y actividades realizados en la instalación productiva y se proyectan cambios para reducir los factores que tienen mayor incidencia en la sostenibilidad. En cada caso, la aplicación sistemática de fases permite proponer alternativas, valorar su idoneidad de acuerdo a las especificaciones del producto y lograr un diseño que tenga en cuenta el impacto ambiental, la rentabilidad económica y el bienestar social.

Palabras clave: Diseño Sostenible, Producto, Etapa De Producción.

\title{
Methodological scheme to design more sustainable products in the production stage
}

\begin{abstract}
Efficiency and sustainability are a challenge for all type of processes and act from the design activity can help to achieve it. In the last decades, Eco-design, focused in the product environmental impacts analysis, has progressively evolved towards methods that analyze simultaneously the three sustainability dimensions: environmental, economic and social.

This work proposes a methodological approach to project more sustainable products in the production stage. This proposal aims to facilitate engineering students the methodological development of theirs projects and researches with sustainability criteria. Three phases are carried out: sustainability assessment applying the Life Cycle Sustainability Assessment (LCSA) methodology, strategies application and comparison of designs. An engineering approach, which defines metrics linked to technical aspects and selects an appropriate set of quantitative indicators to assess each sustainability dimension, is applied. The worker's category is selected to evaluate social aspects.

Methodology is put into practice in two case studies: the manufacture of an isothermal container and the production of milk in a dairy farm. Production data of all materials, processes and activities are analyzed and changes to reduce the greatest impacts are projected. In each case, different design alternatives are proposed and are evaluated taking into account product specifications, environmental impact, economic profitability and social welfare.
\end{abstract}


Keywords: Sustainable Design, Product, Production Stage. 


\section{Introducción}

El término 'desarrollo sostenible' comienza a tener protagonismo en la esfera internacional con la publicación del informe "Our Common Future" (WCED, 1987). El informe analiza y replantea las políticas de desarrollo económico globalizador y pone el foco de atención en el elevado coste medioambiental que conllevan las actividades económicas y los avances sociales. El concepto se reafirma en la Cumbre de la Tierra de Rio de Janeiro (UNCED, 1992) y en las posteriores Conferencias Internacionales que abordan este desafío.

Diferentes autores han analizado este concepto y sus implicaciones. Dalal-Clayton and Bass (2002), consideran que desarrollo sostenible es lograr un desarrollo económico y social sin degradar el ambiente a niveles irreversibles y protegiendo los derechos y oportunidades de las generaciones venideras. Según Bermejo et al. (2010), desarrollo sostenible es el que proporciona el crecimiento necesario para alcanzar la satisfacción de las necesidades humanas esenciales y considera imprescindible mejorar la organización social y garantizar una distribución más equitativa.

De acuerdo con estas definiciones, es necesario actuar en una triple dimensión: social, económica y ambiental (Maxwell et al., 2003). La dimensión ambiental se refiere a la gestión de los recursos del planeta, la dimensión económica al control y distribución de los rendimientos derivados de las actividades industriales y económicas y, la dimensión social, al bienestar de los seres vivos. Se trata de un enfoque basado en el equilibrio entre dimensiones, independientes y conectadas entre sí (Figura 1).

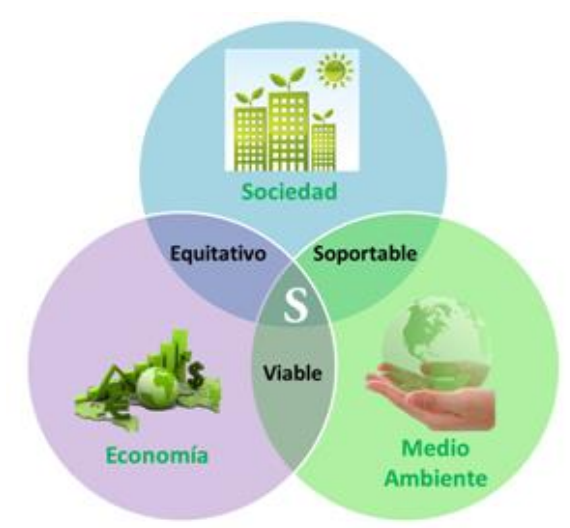

Figura 1. Enfoque de la sostenibilidad: entidades en equilibrio. 
Conscientes de la necesidad de proponer diseños sostenibles, los diseñadores de producto han utilizado a lo largo de las últimas décadas diferentes técnicas y métodos de trabajo. La metodología de Eco-diseño Se basa en el análisis del impacto ambiental de un producto a lo largo de todo su ciclo de vida, desde la obtención de materias primas hasta su tratamiento final, teniendo en cuenta, además, todos los requerimientos propios de la utilización de ese producto en un contexto determinado. Trabajos como los de Keoleian and Menerey, (1993) y Brezet and van Hemel, (1997) exponen las bases del Eco-diseño. Se basan en la realización de tres tareas esenciales: investigar el producto, evaluar el impacto ambiental y plantear estrategias de mejora.

Realizar Eco-diseño requiere la utilización de tres tipos principales de herramientas denominadas, respectivamente, de evaluación ambiental, de mejora ambiental y de integración de requerimientos. La evaluación de impactos ambientales se puede realizar a través de la metodología "Life Cycle Assessment" (LCA). Es un procedimiento normalizado (ISO-14040, 2006a, 2006b) que se compone de cuatro fases principales: i) Definición de objetivos y alcance; ii) Análisis de inventario; iii) Evaluación del impacto; iv) Interpretación de resultados. La aplicación de estrategias de mejora ambiental se basa habitualmente en una serie de principios y reglas que identifican acciones potenciales de mejora. Entre ellas, la denominada rueda de estrategias de diseño para el ciclo de vida o rueda de LiDS (Brezet y Van Hemel, 1997) considera 8 estrategias principales: seleccionar materiales de bajo impacto, reducir materiales, optimizar las técnicas de producción, seleccionar sistemas de comercialización eficientes, reducir el impacto durante el uso del producto, optimizar el tiempo de vida inicial, optimizar el sistema de fin de vida y desarrollar nuevos conceptos. Finalmente, un eco-producto debe cumplir las especificaciones iniciales con las que ha sido ideado. Es decir, es necesario equilibrar los requerimientos de tipo ambiental con otros requerimientos propios del producto (Bovea and Pérez-Belis, 2012).

Un amplio número de trabajos se han desarrollado poniendo en práctica procedimientos con un enfoque basado en el ciclo de vida y utilizando herramientas de evaluación de impacto ambiental. En muchos casos se plantea la evaluación ambiental de un producto con diferentes variantes o alternativas. Lacasa et al., (2016) distinguen tres niveles en el desarrollo de objetivos: a) Evaluación y comparación del impacto en diferentes etapas 
del ciclo de vida; b) Evaluación y comparación del impacto de diferentes alternativas de producto; c) Evaluación del impacto e identificación/aplicación de mejoras en el diseño.

El Eco-diseño se centra en los aspectos técnicos ligados al impacto ambiental de los sistemas y pone una atención limitada en los aspectos humanos. Sin embargo, promover la sostenibilidad significa tener en cuenta principios socio-éticos dentro de un modelo de producción y consumo ecológico y económicamente viable. Con el objetivo de integrar la sostenibilidad en el desarrollo de productos y servicios, Maxwell et al., (2006) elaboran "The Guide for Developing Sustainable Products and Services in industry" recopilando los trabajos más destacados que habían sido realizados hasta ese momento. Como primera etapa del proceso consideran una necesidad funcional y plantean la forma más sostenible de lograr esa función a través de un producto, servicio o sistema producto-servicio.

Varios autores profundizan en el tema del diseño para la sostenibilidad (Crul and Diehl, 2009) y en la evaluación de sus diferentes dimensiones teniendo en cuenta un enfoque de ciclo de vida (Kloepffer, 2007; Finkbeiner et al., 2010). El resultado de sus investigaciones es el desarrollo de un marco de trabajo, denominado LCSA ("Life Cycle Sustainability Assessment"), que analiza y evalúa la sostenibilidad considerando las interconexiones entre sistemas ecológicos y socio-económicos. Para ello combina tres técnicas de evaluación (Figura 2), que se refieren a cada una de las dimensiones que comprende la sostenibilidad: E-LCA ("Environmental Life Cycle Assessment") que se centra en los aspectos medioambientales, LCC ("Life Cycle Costing") que evalúa los aspectos económicos y S-LCA ("Social Life Cycle Assessment") que trata de evaluar el impacto sobre las diferentes organizaciones y agentes sociales. 


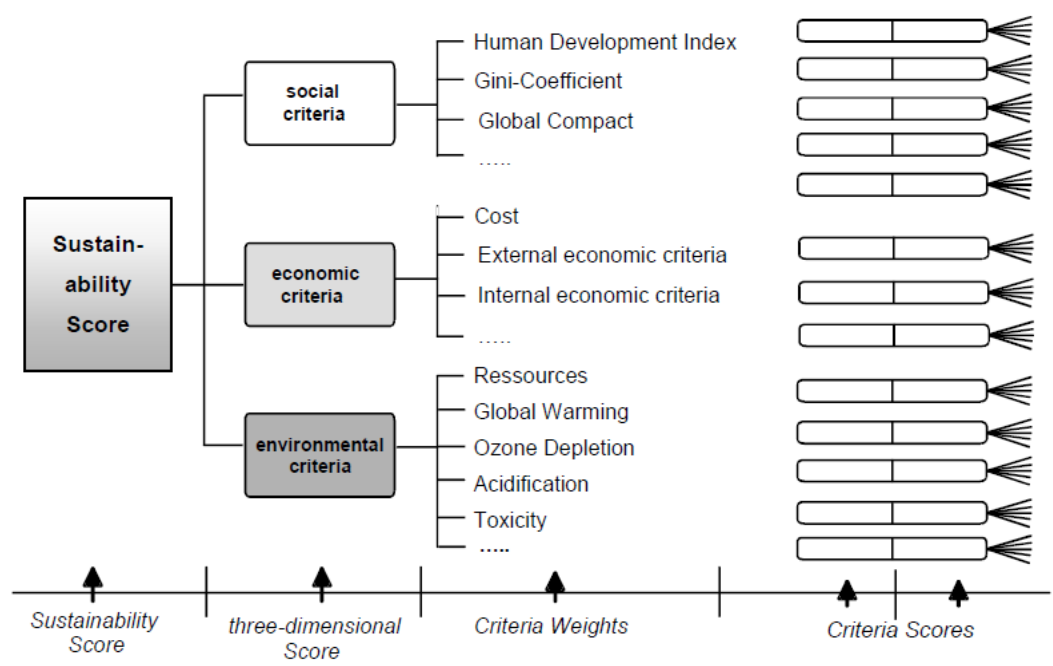

Figura 2. Marco de trabajo LCSA (Finkbeiner et al., 2010).

Este enfoque es expresado como: LCSA $=$ LCA + LCC + S-LCA y permite abordar las tres dimensiones de la sostenibilidad de una forma sistemática, pero su aplicación es todavía limitada y se centra en las dimensiones ambiental y económica. Por tanto, se requiere avanzar en su aplicación y en el desarrollo de métodos de diseño sostenible.

\section{Metodología}

La metodología que se propone en este trabajo pretende mejorar la sostenibilidad de productos actuando en una triple dimensión aplicando LCSA. Se utiliza un enfoque ingenieril que define métricas en paralelo con aspectos técnicos e indicadores cuantitativos adecuados para evaluar la sostenibilidad en cada una de sus dimensiones. Los límites del sistema se establecen en la etapa de producción. Como se muestra en la Figura 3, la metodología se compone de 3 etapas y en cada una de ellas se realizan las tareas que se indican a continuación.

1) Evaluación de la sostenibilidad:

- Se identifica la unidad funcional de acuerdo con el producto analizado.

- Se cuantifican todas las entradas y salidas asociadas al proceso de producción (inventario). Se determina la secuencia de operaciones de fabricación, el consumo de recursos y su coste económico. 
- Se selecciona un conjunto de métricas e indicadores apropiados para evaluar los impactos en cada dimensión.

- Se analizan los resultados y se detectan los factores que generan mayor impacto.

2) Cambios en el diseño de producto:

- Se proponen diferentes alternativas de mejora del producto a partir de la aplicación de diferentes estrategias de sostenibilidad.

- Se comprueba el cumplimiento de las especificaciones iniciales de producto y se excluyen las alternativas que no las cumplen.

- Se selecciona la alternativa más favorable.

- Se evalúa la sostenibilidad del producto rediseñado considerando el nuevo inventario de producción. Se asume que la compañía fabricante dispone de los recursos necesarios (instalaciones, trabajadores y equipamiento industrial) para desarrollar el proceso de fabricación y que éste se lleva a cabo en serie con un elevado volumen de producción.

3) Comparación de diseños:

- Se muestra el comportamiento sostenible de ambos diseños de producto (inicial y rediseño). Dependiendo del carácter de los indicadores utilizados en el análisis, una mejora de la sostenibilidad puede estar asociada al incremento o reducción del indicador.

- Se analizan los resultados y se valoran los cambios producidos. 


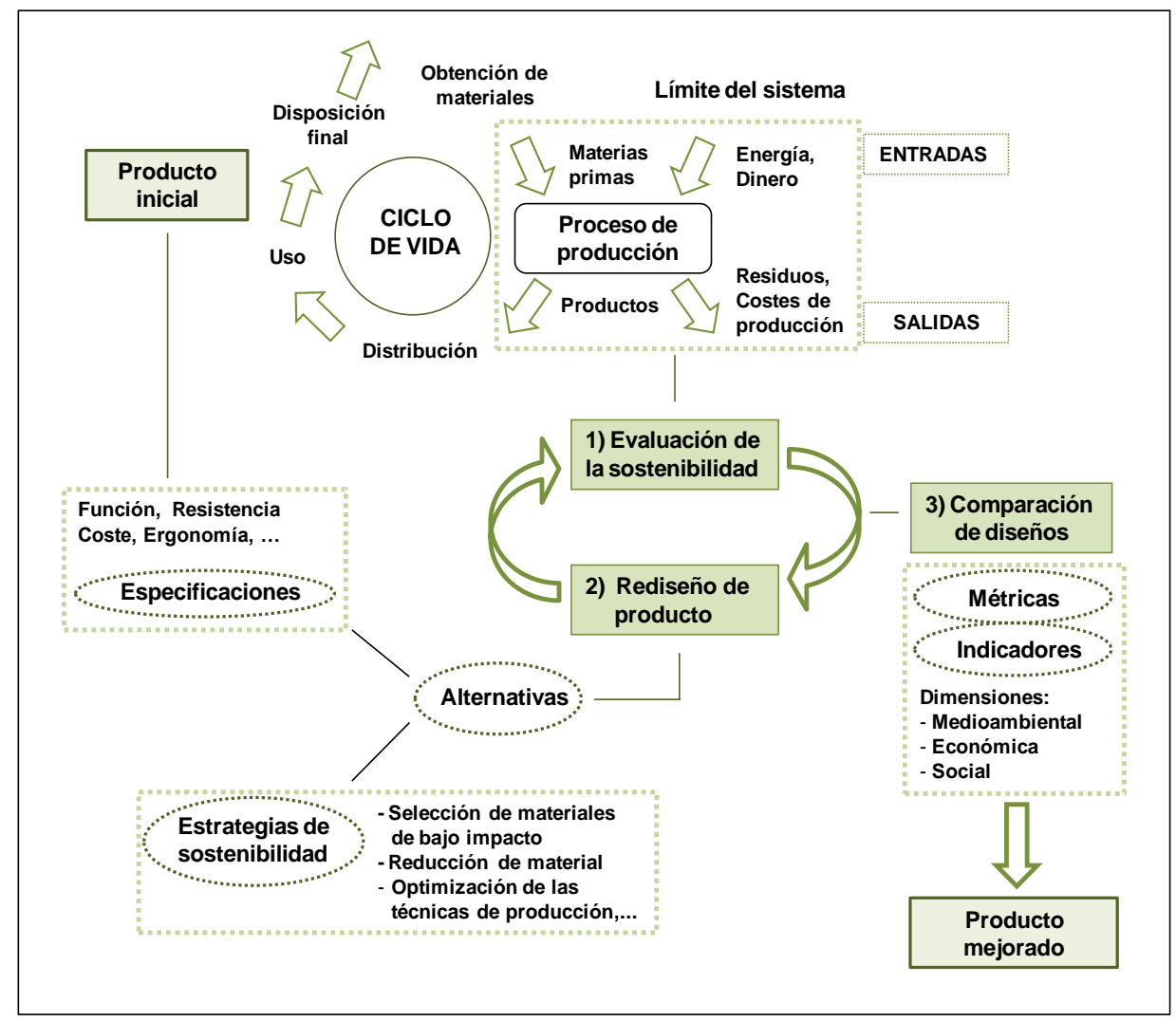

Figura 3. Esquema metodológico para proyectar el desarrollo de productos sostenibles.

En la Tabla 1 se enumeran las métricas e indicadores seleccionados en este trabajo para evaluar la sostenibilidad del producto en la etapa de producción. Las métricas ingenieriles proporcionan información útil a los diseñadores de procesos y son utilizadas para evaluar los indicadores. Los indicadores seleccionados permiten una evaluación cuantitativa y pueden ser utilizados en el estudio de productos muy diversos. Para determinar indicadores ambientales se han consultado bases de datos específicas como Probas (UBA, 2007) o Agrifootprint (Durlinger et al., 2017). De acuerdo con la clasificación de categorías de impacto basada en agentes sociales que establece UNEP/SETAC (2009), en la dimensión social se ha seleccionado para la investigación la categoría de los trabajadores. 


\begin{tabular}{|c|c|}
\hline \multicolumn{2}{|c|}{ Métricas ingenieriles } \\
\hline $\mathrm{M}_{\mathrm{P}}(\mathrm{Kg})$ & $\begin{array}{l}\text { Masa de producto } \\
\text { Es función de la masa de materia prima y la masa de material desechado. }\end{array}$ \\
\hline $\mathrm{M}_{\text {subp }}(\mathrm{Kg})$ & $\begin{array}{l}\text { Masa de subproducto } \\
\text { Masa de productos secundarios generados durante la actividad productiva. }\end{array}$ \\
\hline $\mathrm{V}_{\mathrm{P}}\left(\mathrm{m}^{3}\right)$ & $\begin{array}{l}\text { Volumen de producto } \\
\text { Volumen final de producto terminado y empaquetado. }\end{array}$ \\
\hline $\mathrm{E}_{\mathrm{Pr}}(\mathrm{Kw} \cdot \mathrm{s})$ & $\begin{array}{l}\text { Energía en producción } \\
\text { Consumo de los equipos y máquinas utilizados en operaciones productivas }\end{array}$ \\
\hline$\%$ Res & $\begin{array}{l}\text { Porcentaje de residuo } \\
\text { Material eliminado de la materia prima inicial durante el proceso de fabricación. }\end{array}$ \\
\hline $\mathrm{C}_{\mathrm{Pr}}(€)$ & $\begin{array}{l}\text { Costes de producción } \\
\text { Se obtiene como la suma de los siguientes costes: } \\
\mathrm{C}_{\mathrm{MP}} \text { : Coste de la materia prima utilizada en la fabricación del producto } \\
\mathrm{C}_{\mathrm{MO}} \text { : Coste de la mano de obra empleada en la fabricación del producto } \\
\mathrm{C}_{\mathrm{Cons}} \text { : Coste de los consumibles utilizados } \\
\mathrm{C}_{\text {ind }} \text { : Costes indirectos } \\
\mathrm{C}_{\mathrm{Am}} \text { : Costes de amortización }\end{array}$ \\
\hline $\operatorname{Pr}(\mathrm{ud} / \mathrm{h})$ & $\begin{array}{l}\text { Producción } \\
\text { Unidades de producto fabricadas en un periodo de tiempo }\end{array}$ \\
\hline \multicolumn{2}{|c|}{ Indicadores de sostenibilidad } \\
\hline \multicolumn{2}{|c|}{ Ambientales } \\
\hline $\begin{array}{c}\mathrm{GWP}_{100} \\
\left(\mathrm{Kg} \mathrm{CO}_{2} \text {-eq }\right)\end{array}$ & $\begin{array}{l}\text { Potencial de calentamiento global } \\
\text { Relación entre la contribución a la absorción de calor que resulta de la emisión de } \\
1 \mathrm{Kg} \text { de gas de efecto invernadero y la emisión equivalente de } \mathrm{CO}_{2} \text {, considerando } \\
\text { un horizonte de tiempo de } 100 \text { años. }\end{array}$ \\
\hline Ac & $\begin{array}{l}\text { Acidificación } \\
\text { Reducción del pH debido a emisiones de gases ácidos como los óxidos de } \\
\text { nitrógeno }\left(\mathrm{NO}_{\mathrm{x}}\right) \text { y de azufre }\left(\mathrm{SO}_{\mathrm{x}}\right) \text {. }\end{array}$ \\
\hline $\mathrm{EI} 99(\mathrm{pt})$ & $\begin{array}{l}\text { Eco-indicador } 99 \\
\text { Agrega y expresa de forma ponderada el impacto en diferentes categorías. } \\
\text { Considera tres grupos: salud humana, calidad del ecosistema y recursos. }\end{array}$ \\
\hline \multicolumn{2}{|l|}{ Económicos } \\
\hline VA $(€)$ & $\begin{array}{l}\text { Valor añadido } \\
\text { Representa el beneficio obtenido de la actividad industrial. } \\
\text { Es la diferencia de los ingresos y los costes de producción. }\end{array}$ \\
\hline $\mathrm{EE}(€ / \mathrm{pt})$ & $\begin{array}{l}\text { Eco-eficiencia } \\
\text { Cociente entre VA y EI99. Relaciona el beneficio económico de la actividad } \\
\text { industrial frente al impacto ambiental que genera el desarrollo de esa actividad. }\end{array}$ \\
\hline RSA & $\begin{array}{l}\text { Rentabilidad sobre activos } \\
\text { Cociente entre VA y el activo medio para un periodo dado (normalmente un } \\
\text { ejercicio anual). Representa rentabilidad obtenida respecto a la inversión inicial. }\end{array}$ \\
\hline \multicolumn{2}{|l|}{ Sociales } \\
\hline $\mathrm{T}_{\mathrm{t}}(\mathrm{h})$ & $\begin{array}{l}\text { Tiempo de trabajo } \\
\text { Tiempo promedio de los trabajadores que intervienen en la fabricación de una } \\
\text { unidad de producto. Es función de los tiempos de cada etapa, de la planificación } \\
\text { de tareas y de la asignación de los recursos disponibles en la instalación. }\end{array}$ \\
\hline $\mathrm{S}_{\mathrm{h}}(€ / \mathrm{h})$ & $\begin{array}{l}\text { Salario hora } \\
\text { Salario de los trabajadores que intervienen en la fabricación del producto por } \\
\text { unidad de tiempo trabajada. También expresa el coste de cada hora de trabajo en } \\
\text { el proceso productivo. }\end{array}$ \\
\hline
\end{tabular}

Tabla 1. Métricas ingenieriles e indicadores de sostenibilidad. 


\section{Resultados}

Se presentan dos casos de estudio diferentes. En primer lugar, se analiza un contenedor isotermo utilizado en el almacenamiento y transporte de alimentos. En segundo lugar, se estudia el proceso de obtención de leche en una explotación de ganado vacuno.

\section{Caso 1: Contenedor isotermo}

Este tipo de contenedor se utiliza para el transporte y almacenamiento de alimentos frescos o congelados y tiene una capacidad de 450 1. Sus partes principales se muestran en la Figura 4. Se compone de un bastidor de acero reforzado que aporta resistencia y rigidez, de una pared exterior de poliestireno de alto impacto (HIPS) que permite absorber golpes y de una pared interna realizada en material compuesto de poliestireno y fibra de vidrio, compatible con alimentos. Entre estas dos paredes, un material con bajo coeficiente de transferencia térmica permite la conservación de la cadena de frío de los productos transportados.
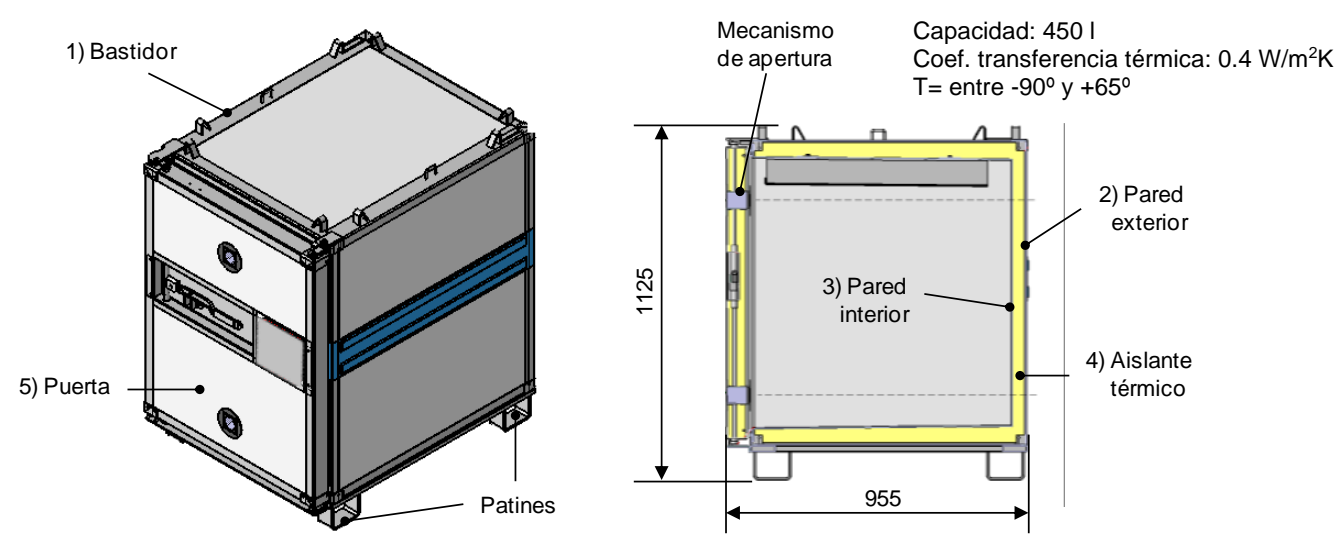

Figura 4. Contenedor isotermo. Componentes y características.

\section{Fase 1: Evaluación de la sostenibilidad}

La producción del contenedor se realiza en serie según un proceso que se muestra de forma resumida en la Figura 5. Se indican las entradas y salidas del proceso, las 
principales operaciones productivas, los tiempos en cada operación y los consumos de energía para fabricar una unidad. Para obtener todos los datos de inventario se ha hecho un seguimiento detallado de todo el proceso productivo en una planta industrial dedicada a su fabricación.

Se puede observar que el ensamblaje es la operación que requiere mayor tiempo de producción, mientras que los procesos de corte por láser y soldadura son las operaciones con mayor consumo de energía. También se indican flujos económicos, considerando los ingresos por la venta del producto y los diferentes costes de producción. Se observa que la materia prima supone la mayor parte del presupuesto de fabricación. En cuanto al porcentaje de material desechado, se comprueba que es muy reducido.

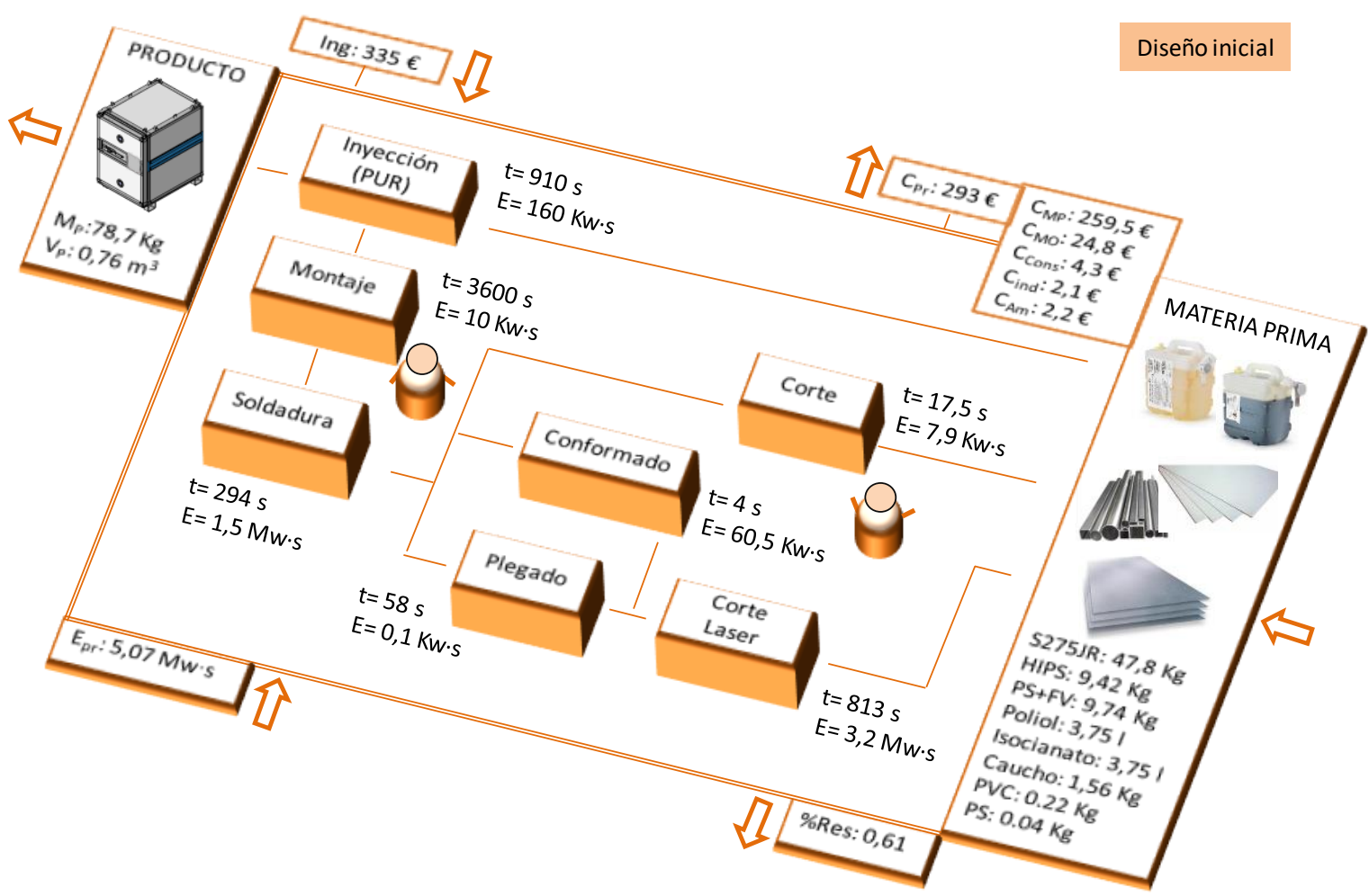

Figure 5. Contenedor isotermo. Línea de producción y flujos de entrada y salida.

La producción promedio de la planta industrial es de 1,2 ud/h. Se considera una producción en serie y una asignación compartida de algunos recursos ya que la instalación fabrica también otro tipo de equipamiento industrial. En particular, para el desarrollo de este producto la instalación dispone de 3 trabajadores en taller y almacén 
con una asignación completa (100\%) y 2 trabajadores en oficina con una asignación del $20 \%$. Las máquinas y equipos de proceso se asignan exclusivamente a la fabricación del contenedor.

A partir del inventario de producción y el cálculo de métricas ingenieriles, se evalúan los indicadores de sostenibilidad. Estos valores se resumen en la Tabla 2. Los resultados muestran que la fabricación de cada contenedor genera la emisión de 120,7 $\mathrm{KgCO}_{2}$-eq. Para unos ingresos/ud. de $335 €$ se obtiene un valor añadido de $42 €$. El tiempo de trabajo promedio empleado en la fabricación de un contenedor es $0,83 \mathrm{~h}$.

\begin{tabular}{|c|c|c|c|c|c|}
\hline \multicolumn{6}{|c|}{ Indicadores de sostenibilidad } \\
\hline \multicolumn{2}{|c|}{ Dimensión medioambiental } & \multicolumn{2}{|c|}{ Dimensión económica } & \multicolumn{2}{c|}{ Dimensión social } \\
\hline $\begin{array}{c}\mathrm{GWP}_{100} \\
\left(\mathrm{Kg} \mathrm{CO}_{2} \text {-eq }\right)\end{array}$ & EI99 $(\mathrm{pt})$ & $\mathrm{VA}(€)$ & $\mathrm{EE}(€ / \mathrm{pt})$ & $\mathrm{T}_{\mathrm{t}}(\mathrm{h})$ & $\mathrm{S}_{\mathrm{h}}(€ / \mathrm{h})$ \\
120,7 & 21,7 & 42 & 1,93 & 0,83 & 29,8 \\
\hline
\end{tabular}

Tabla 2. Contenedor isotermo - Diseño inicial. Indicadores de sostenibilidad.

Con el objetivo de establecer estrategias que mejoren la sostenibilidad del producto en su etapa de producción, se determina la distribución del impacto considerando los materiales utilizados en la fabricación del contenedor.

En la Figura 6a se muestra la distribución del impacto ambiental y en la Figura $6 \mathbf{b}$ la distribución de costes de producción y de tiempos de procesado (sin considerar tiempos de montaje). Se puede observar que los materiales con mayor incidencia son acero galvanizado S275JR (utilizado en el bastidor) y poliuretano (PUR, utilizado como aislante térmico).

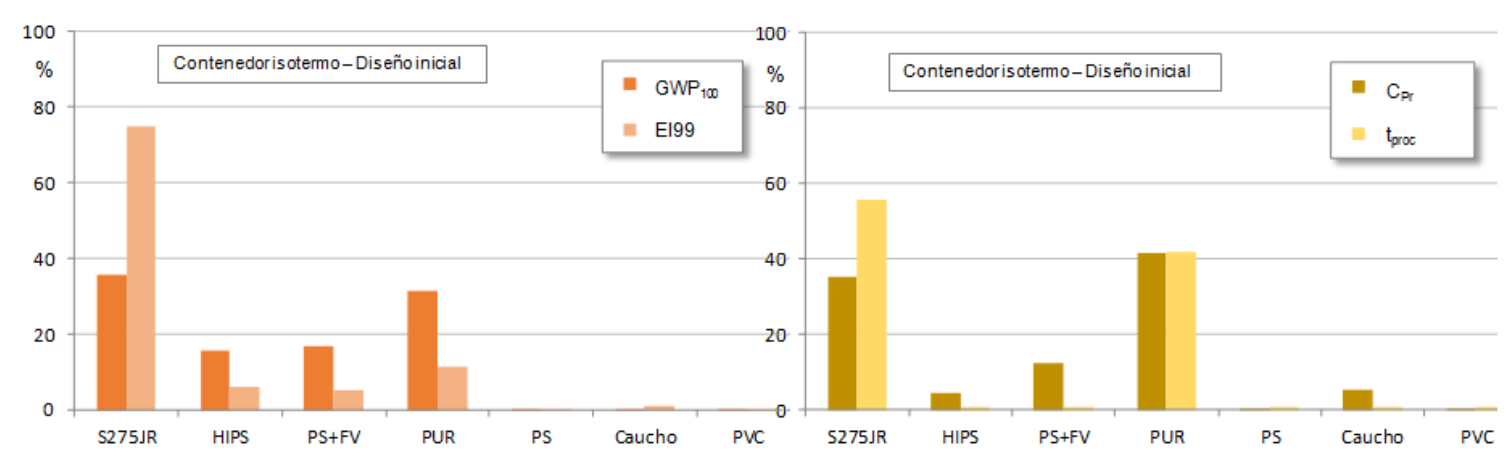

a) Distribución de indicadores ambientales. b) Distribución de costes y tiempos de proceso

Figura 6. Distribución del impacto de los materiales utilizados. 


\section{Fase 2: Cambios en el diseño}

De acuerdo con estos resultados, las estrategias de rediseño se centran en la reducción o sustitución de estos materiales. Además de la capacidad de almacenamiento, se han de mantener una serie de especificaciones en cada una de los subsistemas principales que componen el producto. Estas especificaciones se resumen en la Tabla 3. Junto al material utilizado en el diseño inicial (Di) se plantean diferentes alternativas $\left(\mathrm{A}_{1}, \mathrm{~A}_{2}\right)$, la cantidad de materia prima, el tipo de procesado (en los casos donde se requiera transformación respecto al formato adquirido) y el consumo de energía. El impacto ambiental se evalúa a través del indicador EI99.

\begin{tabular}{|c|c|c|c|c|c|c|c|}
\hline \multirow{2}{*}{\multicolumn{2}{|c|}{ Subsistema }} & \multicolumn{2}{|c|}{ Materia prima } & \multicolumn{2}{|c|}{ Fabricación } & \multirow{3}{*}{$\begin{array}{c}\begin{array}{c}\text { Control de } \\
\text { especificaciones }\end{array} \\
275 \mathrm{MPa} \\
\end{array}$} & \multirow{3}{*}{$\begin{array}{r}\begin{array}{c}\text { EI99 } \\
(\mathbf{m P t})\end{array} \\
13706\end{array}$} \\
\hline & & \multirow{2}{*}{$\begin{array}{l}\text { Material } \\
\text { S275JR g }\end{array}$} & \multirow{2}{*}{$\begin{array}{c}\mathbf{M}_{\text {mat }}(\mathbf{K g}) \\
45,59\end{array}$} & \multirow{2}{*}{$\begin{array}{c}\text { Proceso } \\
\text { Conformado }\end{array}$} & \multirow{2}{*}{$\frac{\mathbf{E}_{\text {mat }}(\mathbf{K w} \cdot \mathbf{s})}{4837,2}$} & & \\
\hline & $\mathrm{D}_{\mathrm{i}}$ & & & & & & \\
\hline 1) Bastidor & $\begin{array}{l}\mathrm{A} 1 \\
\mathrm{~A} 2\end{array}$ & $\begin{array}{l}\text { AISI 304N } \\
\text { Al 6066-O }\end{array}$ & $\begin{array}{l}46,27 \\
16,85\end{array}$ & $\begin{array}{l}\text { Conformado } \\
\text { Conformado }\end{array}$ & $\begin{array}{l}4843,9 \\
1684,9\end{array}$ & $\begin{array}{l}330 \mathrm{MPa} \\
207 \mathrm{MPa}\end{array}$ & $\begin{array}{l}38897 \\
1021,3\end{array}$ \\
\hline \multirow{2}{*}{$\begin{array}{l}\text { 2) Pared } \\
\text { exterior }\end{array}$} & $\mathrm{D}_{\mathrm{i}}$ & HIPS & 9,42 & Comercial & - & $8 \mathrm{KJ} / \mathrm{m}^{2}$ & 1130,4 \\
\hline & $\begin{array}{l}\text { A1 } \\
\text { A2 }\end{array}$ & $\begin{array}{c}\mathrm{PP} \\
\mathrm{PPO}\end{array}$ & $\begin{array}{l}8,57 \\
9,60\end{array}$ & $\begin{array}{l}\text { Comercial } \\
\text { Comercial } \\
\end{array}$ & $\begin{array}{l}- \\
-\end{array}$ & $\begin{array}{l}12 \mathrm{KJ} / \mathrm{m}^{2} \\
15 \mathrm{KJ} / \mathrm{m}^{2}\end{array}$ & $\begin{array}{l}1028,4 \\
1152,1 \\
\end{array}$ \\
\hline \multirow{2}{*}{$\begin{array}{l}\text { 3) Pared } \\
\text { interior }\end{array}$} & $\mathrm{D}_{\mathrm{i}}$ & $\mathrm{PS}+\mathrm{FV}$ & 9,74 & Comercial & - & Compatible & 1003,2 \\
\hline & $\begin{array}{l}\text { A1 } \\
\text { A2 }\end{array}$ & $\begin{array}{c}\text { PS+FL } \\
\text { LDPE+FL }\end{array}$ & $\begin{array}{l}8,91 \\
8,08 \\
\end{array}$ & $\begin{array}{l}\text { Comercial } \\
\text { Comercial }\end{array}$ & $\begin{array}{l}- \\
-\end{array}$ & $\begin{array}{l}\text { Compatible } \\
\text { Compatible }\end{array}$ & $\begin{array}{l}908,3 \\
824,5 \\
\end{array}$ \\
\hline \multirow{2}{*}{$\begin{array}{l}\text { 4) Aislante } \\
\text { térmico }\end{array}$} & $\mathrm{D}_{\mathrm{i}}$ & PUR & 10,21 & Inyección & 160 & $0,034 \mathrm{Kcal} / \mathrm{h} \cdot \mathrm{m} \cdot \mathrm{K}$ & 2145,3 \\
\hline & A1 & Fibra de lino & 9,19 & Comercial & - & $0,033 \mathrm{Kcal} / \mathrm{h} \cdot \mathrm{m} \cdot \mathrm{K}$ & 275,7 \\
\hline \multirow{2}{*}{$\begin{array}{l}\text { 5) Puerta } \\
\text { (guías) }\end{array}$} & $\mathrm{D}_{\mathrm{i}}$ & PVC & 0,22 & Comercial & - & 30 Shore A & 22 \\
\hline & A1 & Caucho & 0,17 & Comercial & - & 70 Shore A & 20,4 \\
\hline
\end{tabular}

Tabla 3. Contenedor isotermo - Rediseño. Utilización de otros materiales.

El rediseño del producto se basa en la selección de las alternativas con menor impacto. En el bastidor se mantiene el acero S275JR como material de fabricación y se aplica una estrategia de reducción de material en piezas no críticas, se obtiene una disminución del $5 \%$ en el indicador EI99. En la pared exterior se proponen como materiales alternativos polipropileno (PP) y poli-óxido de fenileno (PPO). Se selecciona el primero puesto que genera un impacto ambiental ligeramente menor. En la pared interior, se consideran como alternativas poliestireno reforzado con fibra de lino (PS+FL) y polietileno de baja densidad con fibra de lino (LPDE+FL). En los dos casos, se cumple la condición de compatibilidad alimentaria. Se selecciona la segunda opción. Como alternativa al PUR 
utilizado en el aislamiento térmico, se propone emplear fibra de lino. Los dos materiales tienen un coeficiente de conductividad térmica adecuado. La fibra de lino se puede adquirir en planchas comerciales lo que permite reducir el consumo de energía asociado a la inyección de PUR. Finalmente, se propone utilizar caucho en lugar de PVC en las guías de la puerta.

A continuación, se proyecta la producción del contenedor con los cambios señalados. Se realiza un nuevo inventario, determinando tipo, cantidad y coste de materia prima, secuencia de operaciones de fabricación, consumo de energía y tiempos de trabajo. Los indicadores finalmente obtenidos se muestran en la Tabla 4.

\begin{tabular}{|c|c|c|c|c|c|}
\hline \multicolumn{6}{|c|}{ Indicadores de sostenibilidad } \\
\hline \multicolumn{2}{|c|}{ Dimensión medioambiental } & \multicolumn{2}{|c|}{ Dimensión económica } & \multicolumn{2}{|c|}{ Dimensión social } \\
\hline $\begin{array}{c}\mathrm{GWP}_{100} \\
\left(\mathrm{Kg} \mathrm{CO}_{2} \text {-eq }\right)\end{array}$ & $\mathrm{EI} 99(\mathrm{pt})$ & VA $(€)$ & $\mathrm{EE}(€ / \mathrm{pt})$ & $\mathrm{T}_{\mathrm{t}}(\mathrm{h})$ & $\mathrm{S}_{\mathrm{h}}(€ / \mathrm{h})$ \\
\hline 60,8 & 18,2 & 53,5 & 2,93 & 0,7 & 35,3 \\
\hline
\end{tabular}

Tabla 4. Contenedor isotermo - Rediseño. Indicadores de sostenibilidad.

\section{Fase 3: Comparación de diseños}

En la Figura 7 se comparan el producto inicial y el nuevo diseño, utilizando las métricas e indicadores de sostenibilidad previamente definidos.

La variación de métricas se expresa de forma porcentual tomando como referencia el diseño inicial. La optimización de la masa de algunos componentes y la selección de otros materiales permite reducir la masa un 7,5\%, la energía consumida durante el proceso de fabricación un 4,5\% (supone una disminución de $220 \mathrm{Kw} \cdot \mathrm{s}$ ) y los costes de producción un $3,7 \%(11,5 €)$. La fibra de lino tiene un coste más elevado que los componentes del PUR, pero el coste global de la materia prima para constituir el resto de subsistemas del contenedor, es menor. Además, los cambios derivados de la sustitución de PUR por fibra de lino en el aislamiento térmico, suponen una reducción de los tiempos de fabricación y un aumento de la producción en un 7,1\%. 
El análisis de indicadores muestra que la sostenibilidad del contenedor proyectado en el rediseño mejoraría respecto al inicial. Se estima una reducción del impacto ambiental $\left(\mathrm{GWP}_{100}\right.$ disminuye $50,4 \%$ y EI99 disminuye $\left.16,3 \%\right)$, un efecto positivo en los indicadores económicos (VA aumenta $11,5 €$ y EE aumenta $1 € / p t$ ) y una mejora de los indicadores sociales ( $\mathrm{T}_{\mathrm{t}}$ disminuye un $15,7 \%$ y $\mathrm{S}_{\mathrm{h}}$ aumenta un 18,4\%).

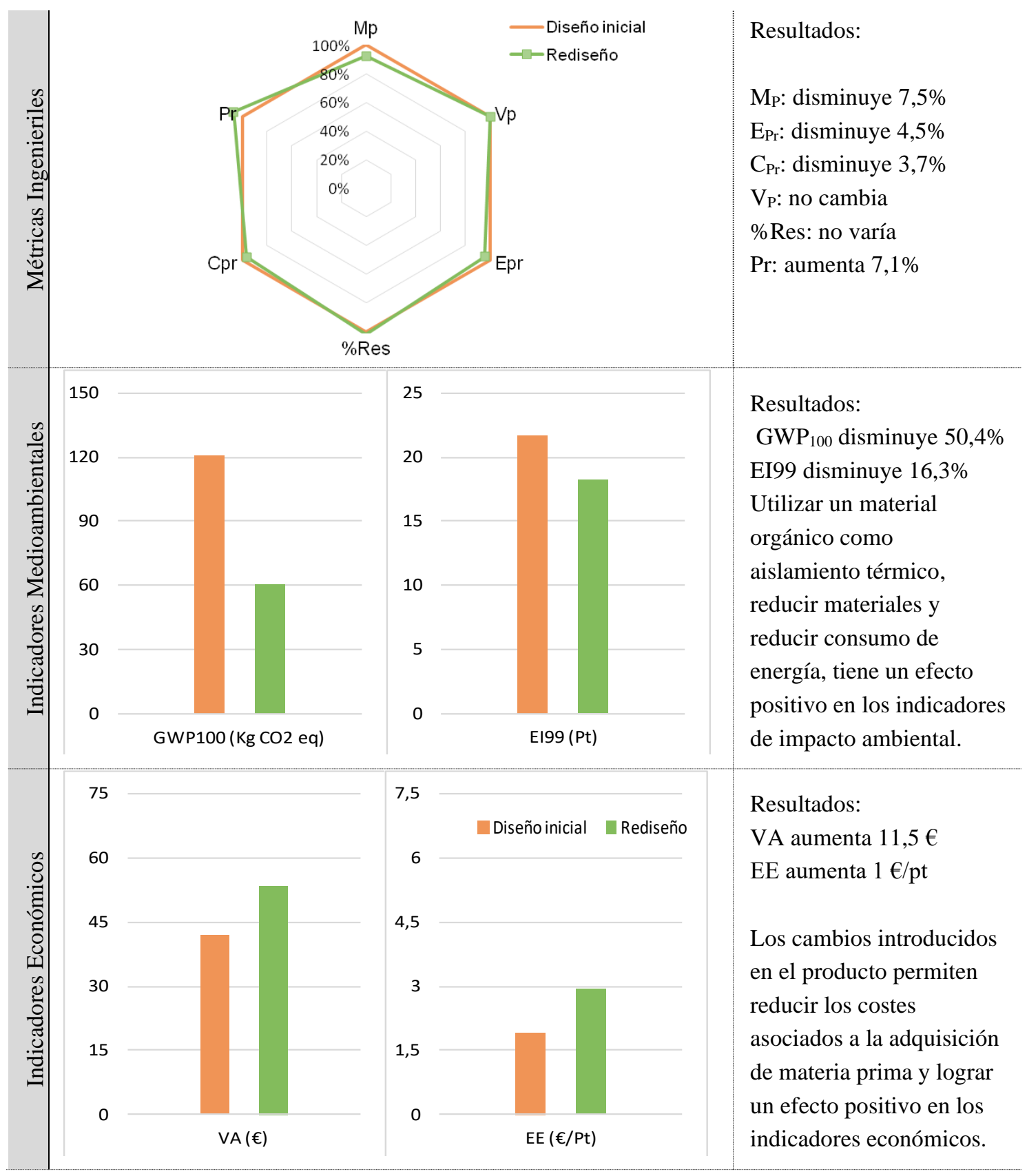




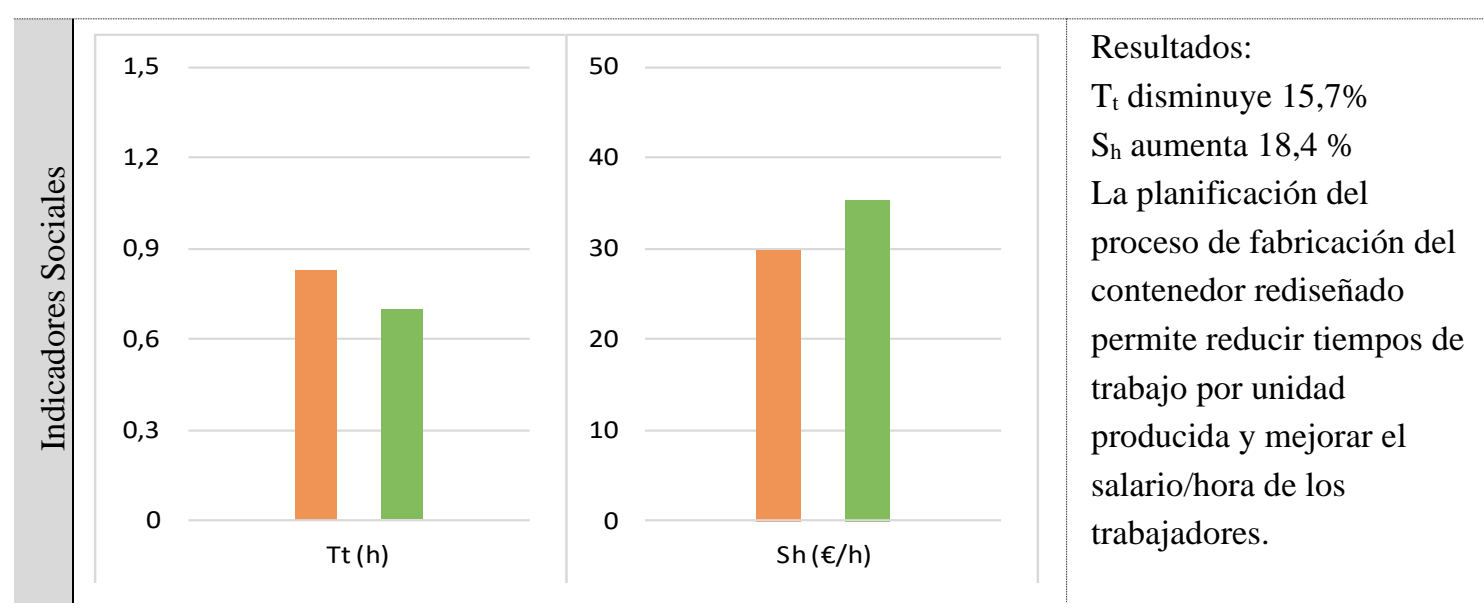

Figura 7. Contenedor isotermo - Comparación de indicadores de sostenibilidad.

\section{Caso 2: Producción de leche}

El estudio se ha llevado a cabo en una explotación láctea de carácter intensivo de tamaño medio-bajo que tiene un promedio de 38 vacas en fase de producción. La alimentación de las vacas influye de forma determinante en la composición y cantidad final de nutrientes de la leche. Una correcta mezcla de alimentos permite obtener leche con la adecuada cantidad de nutrientes. La Fundación Española para el Desarrollo de la Nutrición Animal (FEDNA, 2010) proporciona tablas de composición y valor nutritivo de alimentos que pueden ser consultadas para definir los ingredientes utilizados en la alimentación del ganado.

\section{Fase 1: Evaluación de la sostenibilidad}

Se recopilan las entradas y salidas generadas por las actividades propias de la explotación. Estas actividades consisten principalmente en tareas de alimentación y ordeño de los animales y tareas de mantenimiento y limpieza del recinto. En la Figura 8 se muestra un esquema del proceso productivo. Se indica la cantidad de cada una de las materias primas y las salidas de productos y subproductos. Se muestran los consumos de energía en cada actividad y el consumo global. Además, se muestran los principales flujos económicos intercambiados por el sistema. Todos los datos se expresan por litro de leche producido, que es la unidad funcional utilizada en el análisis. 


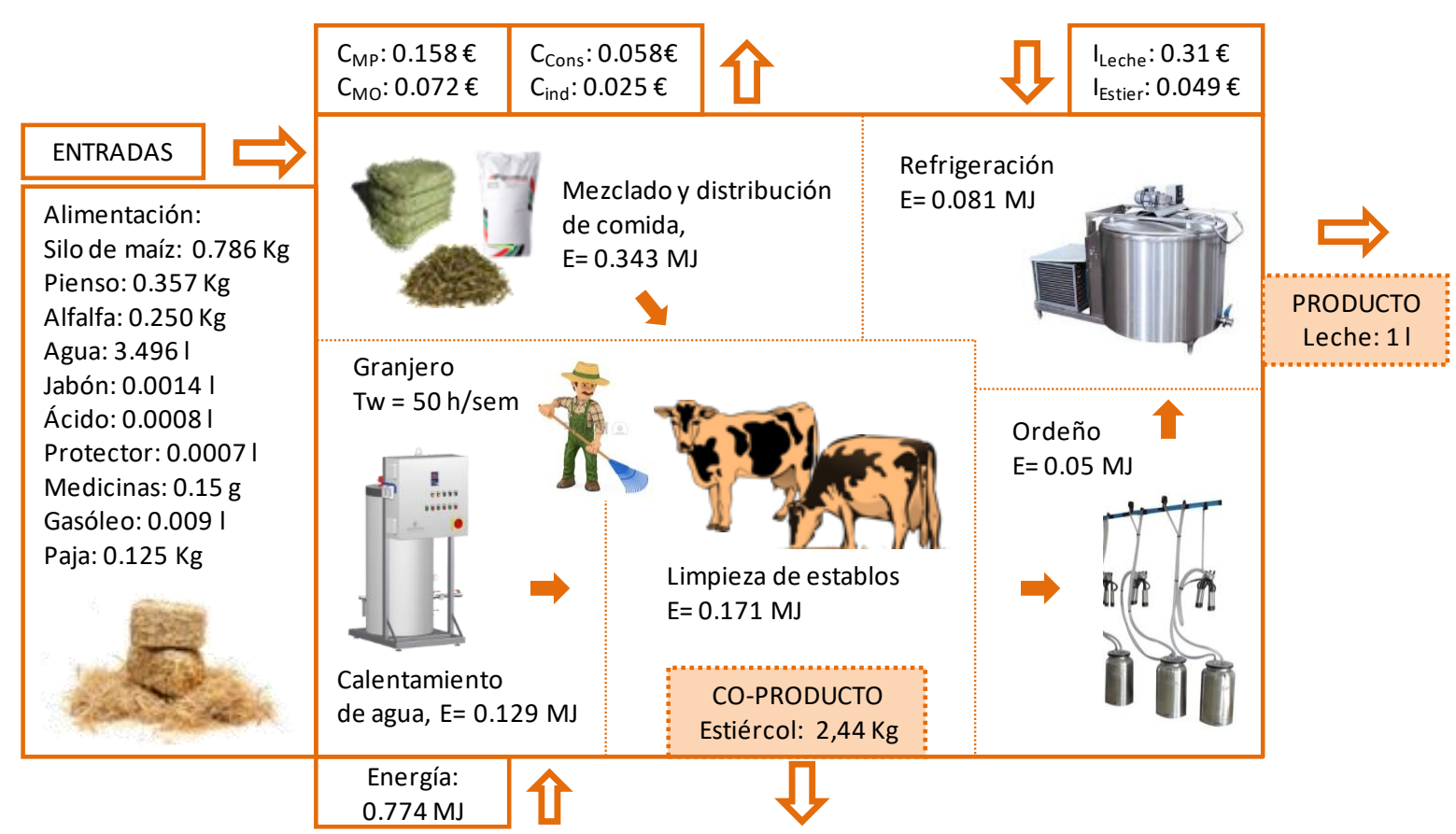

Figura 8. Producción de leche. Esquema del proceso y resumen de inventario.

Las entradas se pueden clasificar en materia prima utilizada en la alimentación de los animales (ensilado de maíz, pienso, alfalfa y agua); productos para la limpieza de los equipos de extracción y almacenamiento de la leche (agua, jabón, ácido y protector), medicamentos, paja para el acondicionamiento de la instalación y gasóleo para el funcionamiento de los tractores.

Cada animal en fase de producción requiere por día, en torno a $40 \mathrm{Kg}$ de comida y entre 80 y 100 litros de agua, dependiendo de la estación del año. En limpieza se emplea agua acompañada de un detergente y un ácido de desinfección específicos. Además, dentro de las entradas al sistema, se contabiliza el consumo de energía en equipos (máquina de ordeño, tanque de almacenamiento y refrigeración de leche y calentadores de agua). Para obtener un litro de leche se requieren $1,393 \mathrm{Kg}$ de alimento y 3,496 1 de agua (3,214 1 son para consumo y 0,282 1 son utilizados en procesos de limpieza). La explotación láctea requiere para su funcionamiento 0,774 MJ de energía y los costes de producción son $0,345 € / 1$.

Como salidas del proceso, se obtiene leche (producto principal) y estiércol (2,44 Kg/l) como subproducto. La producción diaria promedio de leche es de 28 litros por vaca. Se realizan dos ordeños y es una de las actividades a las que el ganadero tiene que dedicar 
más tiempo, ya que requiere la entrada, colocación y retirada de cada animal. El estiércol se obtiene tras la limpieza de la zona de estancia de las vacas. Los ingresos de la explotación no solo proceden de la obtención de leche (el precio establecido es de 0,31 €/1 según Mapama, 2018). También de la recogida y venta de estiércol $(0,02 € / \mathrm{kg})$ y de la comercialización de animales, tanto vacas como animales jóvenes no destinados a la producción de leche. Se debe tener en cuenta, además, que este tipo de explotaciones reciben una subvención por parte de la administración $(0,015 € / 1)$.

Una vez realizado el inventario, se calculan los indicadores de sostenibilidad que caracterizan el proceso inicial de obtención de leche. Se ha considerado adecuado caracterizar el impacto ambiental a través de los indicadores $\mathrm{GWP}_{100}$ y Acidificación (Ac), que son habitualmente utilizados en este sector. Por otra parte, se utilizan VA y RSA como indicadores económicos de la sostenibilidad. Los resultados se muestran en la Tabla 5. Obtener un litro de leche genera unas emisiones de 0,643 $\mathrm{Kg} \mathrm{CO}_{2}$-eq y $0,007 \mathrm{~g} \mathrm{SO}_{2}$ eq. De acuerdo con unos ingresos de $0,428 €$ por litro de leche producido, se obtiene un valor añadido de $0,054 €$ y una rentabilidad sobre activos del 0,069. El tiempo de trabajo empleado en la obtención de un litro de leche es 0,0066 h y el salario/hora es $10,91 € / h$.

\begin{tabular}{|c|c|c|c|c|c|}
\hline \multicolumn{6}{|c|}{ Indicadores de sostenibilidad } \\
\hline \multicolumn{2}{|c|}{ Dimensión medioambiental } & \multicolumn{2}{|c|}{ Dimensión económica } & \multicolumn{2}{|c|}{ Dimensión social } \\
\hline $\begin{array}{c}\mathrm{GWP}_{100} \\
\left(\mathrm{Kg} \mathrm{CO}_{2} \text {-eq) }\right.\end{array}$ & $\begin{array}{c}\mathrm{Ac} \\
(\mathrm{g} \mathrm{SO} 2-e q)\end{array}$ & VA $(€)$ & RSA & $\mathrm{T}_{\mathrm{t}}(\mathrm{h})$ & $\mathrm{S}_{\mathrm{h}}(€ / \mathrm{h})$ \\
\hline 0,643 & 0,007 & 0,054 & 0,069 & 0,0066 & 10,91 \\
\hline
\end{tabular}

Tabla 5. Producción de leche. Indicadores iniciales de sostenibilidad.

A continuación, se analizan los factores de impacto y se determinan cuáles son los que tienen mayor incidencia. En la Figura 9a se muestra la distribución del GWP $\mathrm{F}_{100}$ y de los costes de producción considerando los materiales y consumibles utilizados. En la Figura 9b se muestra la distribución de tiempos y costes asociados a las actividades productivas. La materia prima destinada a la alimentación tiene un elevado impacto ambiental y, en particular, el ensilado de maíz (42\%) y la alfalfa (24\%) son los componentes con mayor contribución. Dentro de los costes de adquisición de materiales y consumibles, el pienso con un 53\%, es el que tiene una contribución más destacada. 


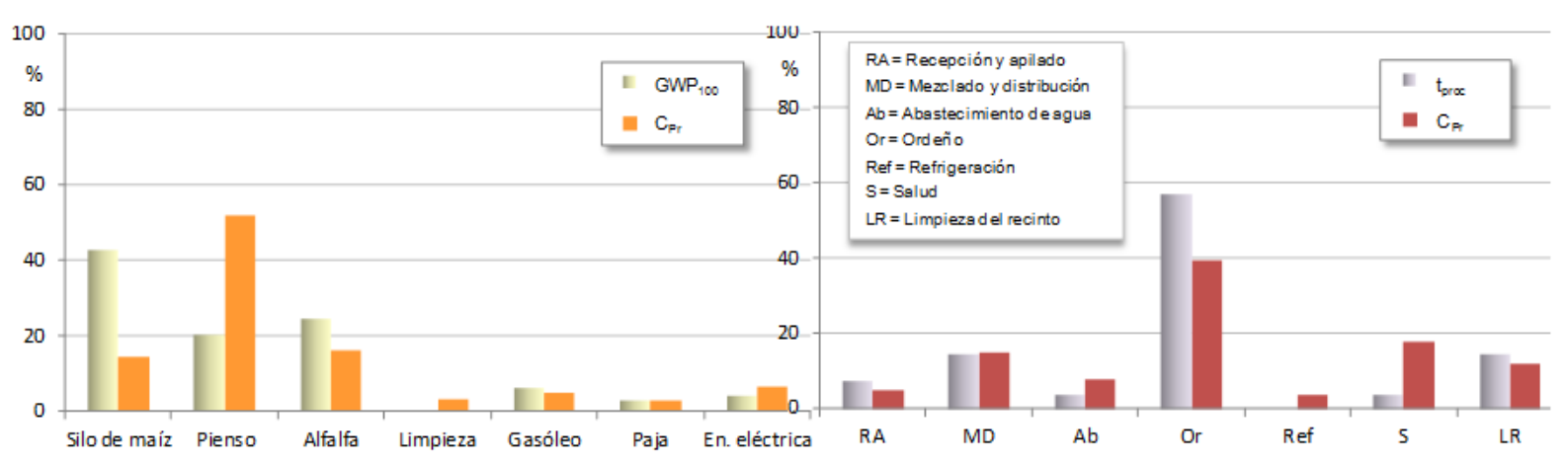

a) Impactos por el consumo de materiales. b) Impactos por el desarrollo de actividades.

Figura 9. Distribución del impacto debido a diferentes factores.

Respecto a las actividades productivas, el ordeño es la que requiere más tiempo y la que genera los costes más elevados (debido a los costes asociados a mano de obra). Las actividades de distribución de comida y de limpieza del recinto tienen un impacto relativo en torno al 15\%. En los costes de actividades relacionadas con la salud se incluyen medicamentos y visitas regulares del veterinario.

\section{Fase 2 - Cambios en el diseño}

A partir de los resultados obtenidos en el análisis previo, las estrategias de rediseño se centran en disminuir el impacto de la materia prima utilizada en alimentación y en reducir el tiempo dedicado a las tareas de ordeño. Para reducir el tiempo de ordeño se plantea la incorporación de otro trabajador con una asignación del 50\%. Para reducir el impacto de la materia prima se recurre al asesoramiento de un nutricionista y se plantean diferentes alternativas de alimentación que aporten a las vacas los nutrientes adecuados. Los valores recomendados se muestran en la Tabla 6.

\begin{tabular}{|l|c|c|c|}
\hline Nutrientes & Cantidad & Unidad & Total $(\mathbf{K g})$ \\
\hline Materia seca & $21-25$ & $\mathrm{Kg}$ & $21-25$ \\
\hline Fibra detergente ácida & $25,5-30,5$ & $\%$ de MS & $5,3-7,6$ \\
\hline Fibra detergente neutra & $43-48$ & $\%$ de MS & $9-12$ \\
\hline Proteína bruta & $16-19$ & $\%$ de MS & $3,4-4,8$ \\
\hline Proteína no degradable & $43-45$ & $\%$ de MS & $9-11,25$ \\
\hline Calcio $(\mathrm{Ca})$ & $1,00-1,10$ & $\%$ de MS & $0,21-0,27$ \\
\hline Magnesio $(\mathrm{Mg})$ & $0,34-0,38$ & $\%$ de MS & $0,07-0,10$ \\
\hline Fosforo $(\mathrm{P})$ & $1,55-1,60$ & $\%$ de MS & $0,33-0,40$ \\
\hline Sodio $(\mathrm{Na})$ & $0,22-0,26$ & $\%$ de MS & $0,05-0,07$ \\
\hline
\end{tabular}

Tabla 6. Alimentación de las vacas - Cantidad de nutrientes recomendados por vaca y día. 
Se hicieron dos propuestas que contemplan dos mezclas de comida diferentes. En la primera alternativa se propone un cambio de ensilado, optando por uno de cebada en lugar de maíz y manteniendo las mismas proporciones en los alimentos que forman la mezcla. En la segunda alternativa, se propone sustituir el ensilado de maíz por otro de paja de cebada y modificar las proporciones de alimentos para equilibrar los nutrientes aportados.

En la Tabla 7 se indican para cada una de las mezclas, los alimentos que la componen, la cantidad que se proporciona a las vacas para obtener un litro de leche y el impacto ambiental generado $\left(\mathrm{GWP}_{100}\right.$ y $\left.\mathrm{Ac}\right)$ por cada alimento individual y cada alternativa de alimentación. En la segunda alternativa, la cantidad total de alimento es menor y genera también un impacto ambiental menor. Puesto que la cantidad de alimento disminuye, algunas actividades del proceso productivo, como las de transporte, mezclado y distribución de la comida, cuyo consumo de energía depende de la masa que se ha de manipular, pueden reducir también el consumo. No obstante, los costes del pienso y de la alfalfa se incrementan $(0,03 € / 1$ en el coste de materia prima) y supone un incremento significativo de los costes de producción globales. Finalmente, esta alternativa de alimentación se implementa en el proceso productivo durante un periodo de 6 meses.

\begin{tabular}{|c|c|c|c|c|}
\hline $\begin{array}{l}\text { Mezclas para } \\
\text { alimentación }\end{array}$ & Componente & $\begin{array}{c}\text { Cantidad } \\
(\mathrm{Kg})\end{array}$ & $\begin{array}{c}\text { GWP }_{100} \\
\left(\mathrm{Kg} \mathrm{CO}_{2} \text {-eq) }\right.\end{array}$ & $\begin{array}{c}\mathrm{Ac} \\
\left(\mathrm{g} \mathrm{SO}_{2} \text {-eq) }\right.\end{array}$ \\
\hline \multirow{4}{*}{ Diseño inicial } & Ensilado de maíz & 0,786 & 0,275 & 0,0044 \\
\hline & Pienso & 0,357 & 0,129 & 0,0008 \\
\hline & Alfalfa & 0,251 & 0,156 & 0,0017 \\
\hline & Total & 1,394 & 0,560 & 0,0069 \\
\hline \multirow{4}{*}{ Alternativa 1} & Ensilado de cebada & 0,786 & 0,418 & 0,0045 \\
\hline & Pienso & 0,357 & 0,129 & 0,0008 \\
\hline & Alfalfa & 0,251 & 0,156 & 0,0017 \\
\hline & Total & 1,394 & 0,703 & 0,0070 \\
\hline \multirow{4}{*}{ Alternativa 2} & Paja & 0,143 & 0,021 & 0,0001 \\
\hline & Pienso & 0,500 & 0,180 & 0,0012 \\
\hline & Alfalfa & 0,357 & 0,223 & 0,0024 \\
\hline & Total & 1 & 0,424 & 0,0037 \\
\hline
\end{tabular}

Tabla 7. Obtención de leche. Alternativas de alimentación. 
A continuación, tras los cambios introducidos se realiza un nuevo inventario de producción y se calculan las métricas e indicadores de sostenibilidad correspondientes al rediseño. Para obtener un litro de leche se necesita $1 \mathrm{Kg}$ de alimento y 3,496 litros de agua. Se consumen $686,8 \mathrm{Kw} \cdot \mathrm{s}$ de energía y se genera 2,6 Kg de estiércol. Los ingresos por venta de estiércol se incrementan un 6\%. El resto de ingresos no se modifican. Los costes de producción aumentan a 0,395€. La producción diaria promedio es 1064 litros/día (28 litros por vaca y día), por tanto, se mantiene igual a la del proceso inicial. Los indicadores finalmente obtenidos se muestran en la Tabla 8.

\begin{tabular}{|c|c|c|c|c|c|}
\hline \multicolumn{9}{|c|}{ Indicadores de sostenibilidad } \\
\hline \multicolumn{2}{|c|}{ Dimensión medioambiental } & \multicolumn{2}{|c|}{ Dimensión económica } & \multicolumn{2}{|c|}{ Dimensión social } \\
$\mathrm{GWP}_{100}$ & $\mathrm{Ac}$ & $\mathrm{VA}(€)$ & $\mathrm{RSA}$ & $\mathrm{T}_{\mathrm{t}}(\mathrm{h})$ & $\mathrm{S}_{\mathrm{h}}(€ / \mathrm{h})$ \\
$\left(\mathrm{Kg} \mathrm{CO}_{2}\right.$-eq $)$ & $\left(\mathrm{g} \mathrm{SO}_{2}\right.$-eq) & $\mathrm{VA}$ & 0,009 & 0,0056 & 16,97 \\
0,5 & 0,004 & 0,007 & 0,009 \\
\hline
\end{tabular}

Tabla 8. Producción de leche. Indicadores de sostenibilidad.

\section{Fase 3. Comparación de diseños}

En la Figura 10 se comparan la producción inicial y final utilizando las métricas e indicadores de sostenibilidad previamente definidos. Los cambios introducidos en la mezcla de materia prima para alimentación suponen un cambio considerable en la masa total de alimento que se reduce un $26,6 \%$. La cantidad de agua no varía siendo en promedio 3,49 1 por litro de leche producido. El consumo de energía en la explotación se reduce un $10,3 \%$ debido a que se reduce el consumo en las actividades de mezclado y distribución de comida (más ligera). La masa de estiércol aumenta un 6\% como consecuencia de que la alimentación contiene un porcentaje más elevado de materia seca. Los costes de producción se incrementan un 14,5\% debido a dos factores, el aumento de los costes de materia prima para alimentación y el de los costes de mano de obra. Finalmente, la producción diaria de leche se mantiene en un promedio de 10641.

Los indicadores ambientales mejoran ( $\mathrm{GWP}_{100}$ disminuye $22 \%$ y Ac disminuye $43 \%$ ). Sin embargo, incrementan notablemente los costes de producción en materia prima (19\%) y en mano de obra. Los ingresos por producción de leche no varían y los de venta de estiércol aumentan ligeramente. En consecuencia, los indicadores económicos 
disminuyen. VA se reduce $0,047 €$ y RSA se reduce 0,06 . Además, la contratación de un trabajador permite reducir el tiempo promedio de trabajo un $15 \%$ y puesto que la mano de obra aumenta, el salario hora también se incrementa un 55\%. Estos resultados muestran que una alimentación basada en ensilado de maíz, pienso y alfalfa tiene un rendimiento económico mayor que una alimentación basada en paja, pienso y alfalfa, pero tiene un impacto ambiental mayor. Por otra parte, el rendimiento económico disminuye si aumenta el número de trabajadores, pero los indicadores sociales mejoran.

Puesto que no se produce una mejora simultánea de los indicadores, para determinar si se logra una mejora global de la sostenibilidad del sistema, será necesario asignar coeficientes de ponderación según la importancia que se le concede a cada dimensión. En la Tabla 9 se muestran cuatro casos diferentes. En el caso 1, todas las dimensiones tienen la misma importancia y en los casos 2, 3 y 4 una de ellas es dominante. Se puede observar que solo en los casos 2 y 4, en las que las dimensiones ambiental y social son consideradas dominantes, se obtiene un proceso de producción de leche más sostenible.

\begin{tabular}{|c|c|c|c|c|c|}
\cline { 3 - 6 } \multicolumn{1}{c|}{} & Caso 1 & Caso 2 & Caso 3 & Caso 4 \\
\hline Indicador & Variación (\%) & \multicolumn{4}{c|}{ Ponderación } \\
\hline $\mathrm{GWP}_{100}$ & 22 & 0,166 & 0,250 & 0,125 & 0,125 \\
\hline $\mathrm{Ac}$ & 43 & 0,166 & 0,250 & 0,125 & 0,125 \\
\hline $\mathrm{VA}$ & -87 & 0,166 & 0,125 & 0,250 & 0,125 \\
\hline $\mathrm{RSA}$ & -87 & 0,166 & 0,125 & 0,250 & 0,125 \\
\hline $\mathrm{T}_{\mathrm{t}}$ & 15 & 0,166 & 0,125 & 0,125 & 0,250 \\
\hline $\mathrm{S}_{\mathrm{h}}$ & 55 & 0,166 & 0,125 & 0,125 & 0,250 \\
\hline & & \multicolumn{4}{|c|}{ Mejora de la sostenibilidad (\%) } \\
\cline { 3 - 6 } & & $\mathbf{- 6 , 5}$ & $\mathbf{3 , 2}$ & $\mathbf{- 2 6 , 6}$ & $\mathbf{3 , 8}$ \\
\cline { 3 - 6 } & & &
\end{tabular}

Tabla 9. Producción de leche. Valoración global de la sostenibilidad. 


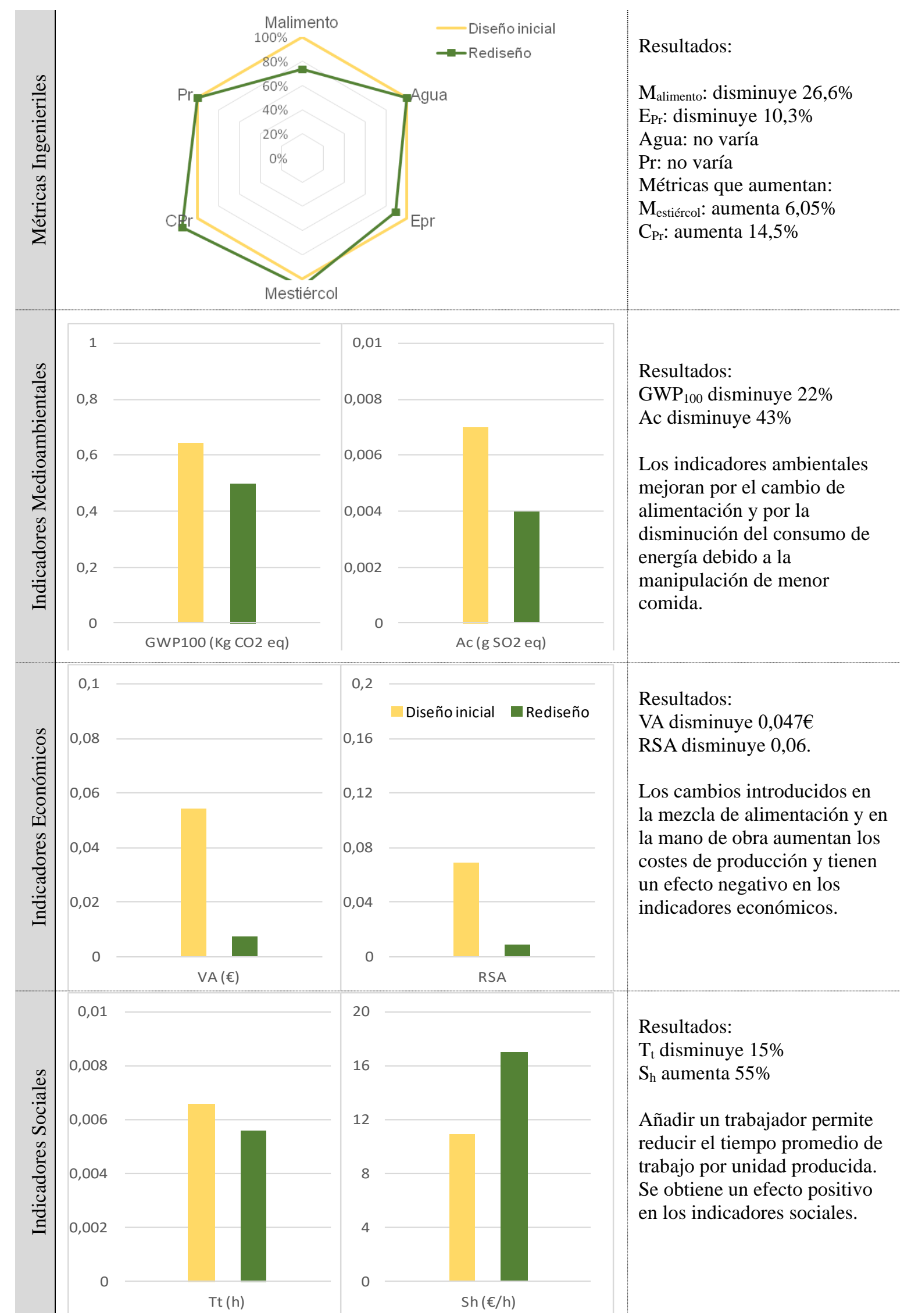

Figura 10. Producción de leche - Comparación de indicadores de sostenibilidad. 


\section{Discusión}

El estudio y evaluación de la sostenibilidad en cada caso, requiere un análisis detallado del producto, su descomposición en partes más simples, que pueden ser subsistemas, subconjuntos, piezas, componentes, materiales, ... con objeto de conocer en detalle su constitución, sus características y especificaciones. El resultado de este análisis permite abordar el inventario de producción. Además, realizar el inventario de producción requiere obtener y organizar todos los datos que caracterizan cada una de las operaciones del proceso productivo, analizar las transformaciones de material, el consumo de energía, la asignación de recursos, el tiempo empleado y el coste económico.

El estudio completo y preciso de producto y proceso productivo permite identificar y seleccionar un conjunto de métricas ingenieriles significativas y evaluar los indicadores de sostenibilidad en cada una de sus dimensiones. Se ha trabajado con un conjunto común de métricas e indicadores en todos los casos. No obstante, se seleccionan en cada caso, los considerados más eficaces para desarrollar la metodología y lograr sus objetivos. Por otra parte, la selección de métricas e indicadores se podría ampliar con el objetivo de realizar un análisis y evaluación más completos y que sean adecuados a las particularidades del producto y a las estrategias aplicadas en el rediseño.

El proceso de mejora del producto se ha basado en la propuesta y selección de alternativas que cumplan las especificaciones convencionales del producto y apliquen estrategias que permitan reducir los focos de impacto. Se comprueba que para plantear estrategias de rediseño eficaces es necesario determinar cuáles son los factores que afectan de forma determinante a la sostenibilidad. En el diseño inicial y en las diferentes alternativas de rediseño, los indicadores ambientales son evaluados considerando aquellas etapas del ciclo de vida que puedan verse comprometidas. De esta forma, se pretende plantear opciones de rediseño válidas y que no se vean rechazadas por no considerar este tipo de enfoque global. 
El objetivo de la sostenibilidad se logra tras un proceso secuencial y sistemático, en el que el desarrollo de cada fase está condicionado por la fase anterior: i) Proyectar una mejora en la sostenibilidad de un producto supone aplicar estrategias adecuadas de diseño; ii) Plantear estrategias de rediseño sostenible efectivas conlleva la detección de los factores más influyentes; iii) Seleccionar una alternativa de rediseño implica comprobar que las especificaciones iniciales del producto se cumplen; iv) Medir la sostenibilidad requiere el análisis de inventario; v) Abordar el inventario de producción requiere el análisis del producto.

El estudio comparativo de diseños se realiza a través del análisis individual de métricas e indicadores de cada dimensión. Si se obtiene una mejora simultánea de todos los indicadores, el sistema mejora de manera global con el nuevo diseño. Si los indicadores muestran diferentes tendencias, es necesario asignar coeficientes de ponderación que establezcan la importancia de cada indicador en la valoración global de la sostenibilidad del sistema y permitan tomar decisiones sobre el comportamiento sostenible del nuevo diseño realizado.

\section{Conclusiones}

Este trabajo contribuye a abordar el desafío de la sostenibilidad de una forma global. Para ello, se propone una metodología que analiza y evalúa impactos en cada una de sus tres dimensiones: ambiental, económica y social y aplica estrategias globales de mejora. En la literatura científica, los estudios de sostenibilidad de producto se limitan a aplicar una metodología de evaluación. En muy pocos casos se aportan alternativas de rediseño, se proyectan soluciones, se evalúan y se comparan con el diseño inicial. La metodología planteada, además de evaluar la sostenibilidad tiene como objetivo mejorarla.

Se han seleccionado y utilizado un conjunto de métricas e indicadores que facilitan el análisis del producto con una perspectiva ingenieril y permiten la evaluación cuantitativa de cada una de las dimensiones de la sostenibilidad en la fase de producción. De esta forma, se contribuye a integrar los requerimientos de sostenibilidad en la metodología proyectual propia de la ingeniería y dentro del ámbito del diseño de 
producto. Para ello, se identifican especificaciones, se analizan factores, se proponen alternativas de diseño y se analizan respecto al diseño inicial.

El esquema metodológico se ha puesto en práctica en dos casos de estudio, comprobando en ambos casos la posibilidad de obtener un producto más sostenible a través de la aplicación de estrategias basadas en reducir los focos de impacto. Se prevé continuar la investigación ampliando el alcance a todo el ciclo de vida del producto e incorporando otros indicadores capaces de cuantificar impactos en un número más elevado de categorías.

\section{Bibliografía}

Bermejo, R., Arto, I., Hoyos, D., Garmendia, E., 2010. Menos es más. Del desarrollo sostenible al decrecimiento sostenible. Cuadernos de trabajo de Hegoa 52.

Bovea, M.D. and Pérez-Belis, V., 2012. A taxonomy of eco-design tools for integrating environmental requirements into the product design process, Journal of Cleaner Production 20:1, 61-71.

Brezet, J.C. and Van Hemel C.G., 1997. Ecodesign: a promising approach to sustainable production and consumption. UNEP, United Nations Publications, Paris.

Crul, M. and Diehl, J.C., 2009. Design for sustainability. A step-by-step approach. UNEP, United Nations Publications, Paris.

Dalal-Clayton, D.B. and Bass, S., 2002. Sustainable development strategies: A resource book. Earthscan publications Ltd., England.

Durlinger, B., Koukouna, E., Broekema, R., van Paassen, M., Scholten, J., 2017. Agrifootprint 3.0 Gouda, NL.

FEDNA, 2010. Fundación Española para el Desarrollo de la Nutrición Animal.

Disponible en: www.fundacionfedna.org/tablas-fedna-composicion-alimentos-valornutritivo

Finkbeiner, M., Schau, E.M., Lehmann, A., Traverso, M., 2010. Towards life cycle sustainability assessment. Sustainability 2, 3309 - 3322. 
ISO, 2006a. ISO 14040 International Standard. In: Environmental management - Life cycle assessment - Principles and framework. International Organisation, Geneva, Switzerland.

ISO, 2006b. ISO 14040 International Standard. In: Environmental management - Life cycle assessment - Requirements and Guidelines. International Organisation, Geneva, Switzerland.

Keoleian, G., Menerey, D., 1993. Life Cycle Design Guidance Manual, EPA/600/R92/226, Risk Reduction Engineering Laboratory, United States Environment Protection Agency.

Kloepffer, W., 2007. Life-cycle based sustainability assessments as part of LCM. In Proceedings of the 3rd International Conference on Life Cycle Management, Zurich, Switzerland, 27-29 August, 2007.

Lacasa, E., Santolaya, J.L., Biedermann, A., 2016. Obtaining sustainable production from the product design analysis. Journal of Cleaner Production 139, 706-716.

MAPAMA, 2018. Ministerio de Agricultura, Pesca, Alimentación y Medio Ambiente.

Disponible en: www.mapama.gob.es/es/alimentacion/servicios/observatorio-de-precios-delos-alimentos/estudios-e-informes/

Maxwell, D., Van der Vorst, R., 2003. Developing sustainable products and services. Journal of Cleaner Production 11, 883-895.

Maxwell, D., Sheate, W., Van der Vorst, R., 2006. Functional and systems aspects of the sustainable product and service development approach for industry. Journal of Cleaner Production 14, 1466-1479.

UNEP/SETAC, 2009. Guidelines for Social Life Cycle Assessment of Products. United Nations Environment Programme, Paris.

UBA, 2007. Umweltbundesamt (UBA). German Environmental Protection Agency.

UNCED, Agenda 21, United Nations Conference on Environment and Development, Rio de Janeiro, June 1992.

WCED (World Commission on Environment and Development), 1987. Our common future. Oxford University Press. 